\section{Lack of activation-induced cytidine deaminase expression in in situ follicular neoplasia}

The immunoglobulin heavy chain $(I G H)$ genes undergo class switch recombination (CSR) and somatic hypermutation (SHM) to produce a diverse repertoire of high affinity antibodies. CSR and SHM occur in mature B lymphocytes in the highly specialized microenvironment of germinal centers (GC) and depend on activation-induced cytidine deaminase (AID), an enzyme present in germinal center B cells. AID mediates deamination of cytosine $(C)$ to uracil $(\mathrm{U})$ at cytosine:guanine $(\mathrm{C}: \mathrm{G})$ base pairs to generate uracil:guanine $(\mathrm{U}: \mathrm{G})$ mismatch, and double-strand DNA breaks (DSB) into switch $(S)$ region sequences of $I G H$. This process introduces point mutations at a high frequency in $I G H V$ and $I G L V$ genes, allowing for affinity maturation of the antibody response, and replaces the heavy chain constant region of the antibody from the default IgM to IgG, IgA or IgE isotypes. ${ }^{1}$

In addition to $\mathrm{IGH}$, endogenous AID affects numerous genes across the B-cell genome but at much lower levels than the immunoglobulin loci. Off target AID activity during the germinal center reaction contributes to lymphomagenesis. ${ }^{2}$ In malignancies of mature B-cell origin, recurrent translocations often arise as a result of DSB within the IGH switch region and a partner gene. These translocations arise as a result of aberrant AID mediated $\mathrm{CSR}^{2}$ Mutations in various oncogenes implicated in the pathogenesis of B-cell lymphomas like BCL6, PAX5, MYC and $B C L 2$ share features similar to immunoglobulin gene mutations suggesting a role for aberrant $S H M$.

Based on RNA expression data, AID appears to be selectively expressed in GC B cells and GC-derived malignancies. ${ }^{3}$ Follicular lymphoma (FL) originates from GC B cells and shows heterogeneity in the SHM pattern, suggesting the possible heterogeneous expression of AID in this entity. The reported expression of AID in FL by immunohistochemistry (IHC) varies from $25 \%$ to $100 \%$, acknowledging that some of this variation may be due to technical and definitional differences. ${ }^{3,4,5}$ More recently, Scherer and colleagues demonstrated that AID messenger RNA (mRNA) and protein expression were highly correlated in $\mathrm{FL}$ by quantitative reverse transcriptase polymerase chain reaction (RT-PCR) and IHC, respectively. However, there was great variability with $38 \%$ of cases showing less than ten IHC-positive cells/high power field (hpf). ${ }^{6}$ This translates to $1 \%$ or less of cells in a hpf of small lymphocytes. Interestingly, duodenal-type FL (DFL) lacks AID expression, which has been postulated to be related to its extremely indolent clinical behavior. Immunophenotypically, DFL cells typically strongly co-express CD10 and BCL2 proteins. In situ follicular neoplasia (ISFN) is characterized by partial or total colonization of reactive germinal centers by $\mathrm{BCL} 2^{+} / \mathrm{CD} 10^{+}$coexpressing B cells harboring IGH-BCL2 fusions in an otherwise architecturally preserved lymph node. BCL2 and CD10 IHC staining is characteristically intense and is higher than that seen in systemic FL cells, much like DFL. Given all the overlapping characteristics of these entities, we explored AID expression in ISFN.

Institutional pathology archives (2002-2017) were searched for cases of ISFN and re-reviewed to confirm diagnosis. We identified 16 patients with ISFN (six male, ten female) with adequate tissue for study. The median age was 66 years (range, $50-83$ years) at the time of diagnosis. Additional clinical information was obtained by review of the medical records and was available for 13 patients. The ISFN was discovered incidentally in all the cases, during pathologic review of biopsies performed for a variety of clinical indications. Ten patients had other hematolymphoid or non-hematolymphoid neoplasms at some point in time. Two patients had a history of preceding FL, and one patient had concurrent FL at another anatomic site. Of the non-FL patients, none developed subsequent FL on median follow-up of 43.5 months (range, 13-97 months). 11 patients were alive at last the follow-up, one was lost to follow-up and one patient died from complications of chronic myelomonocytic leukemia and related treatment. The clinicopathologic features are summarized in the Online Supplementary Table S1.

Morphologically, the lymphoid and immune architecture was preserved in all ISFN cases, with a few follicles demonstrating BCL2 highly overexpressing GC B cells. Initially, six ISFN cases were subjected to immunohistochemical staining with AID which suggested lack of staining in ISFN cells compared to the expression in reac-

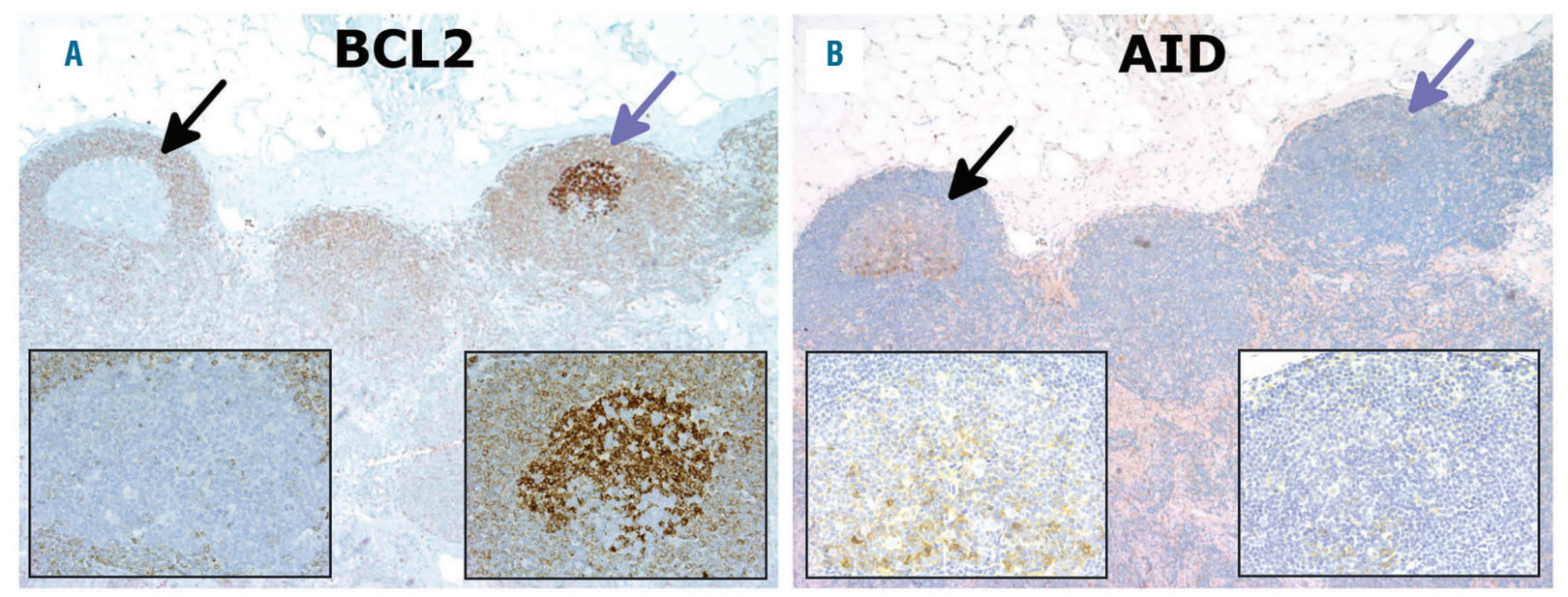

Figure 1. BCL2 and AID immunohistochemistry. (A) Intense BCL2 staining in follicle involved by in situ follicular neoplasia (ISFN) (blue arrow) and negative BCL2 staining in reactive follicle (black arrow). (B) In contrast, ISFN follicle (blue arrow) lacks activation-induced cytidine deaminase (AID), and reactive follicle (black arrow) is positive for AID. 
BCL2
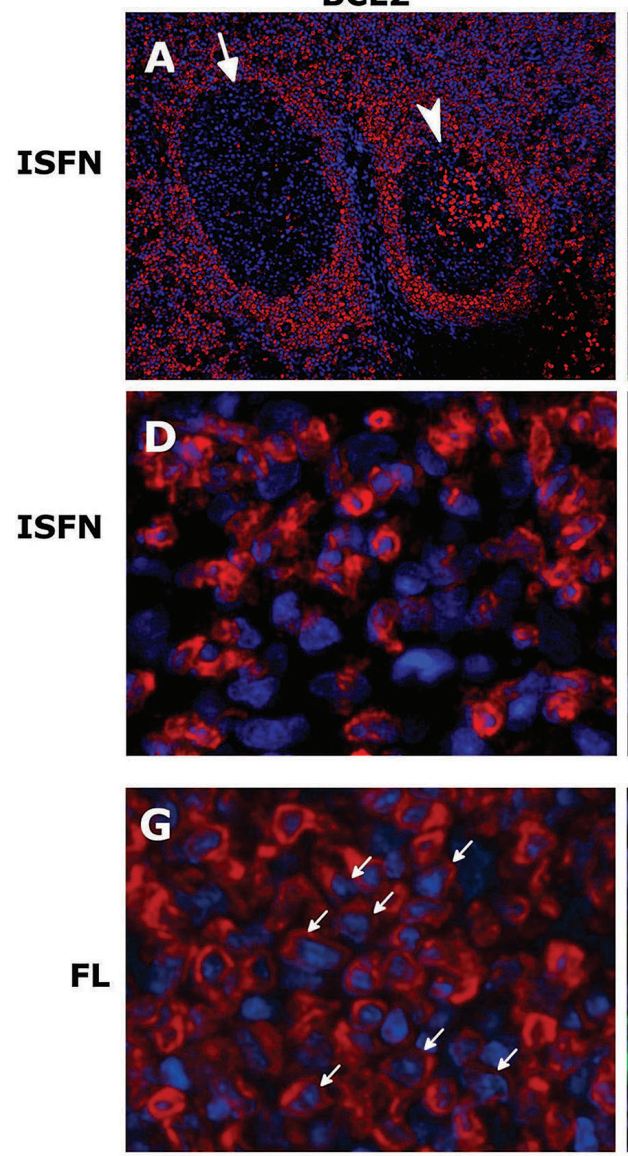

AID
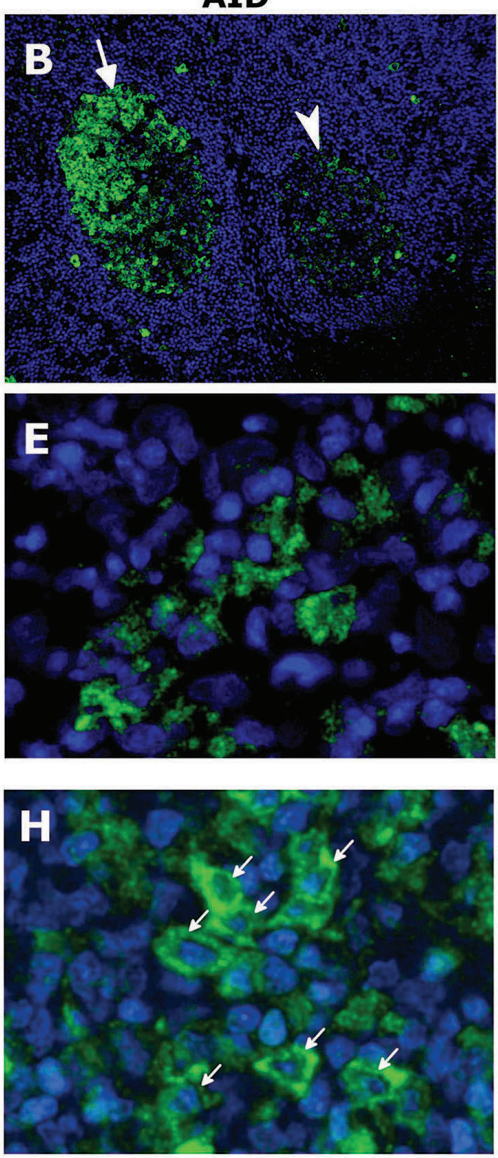

AID/BCL2
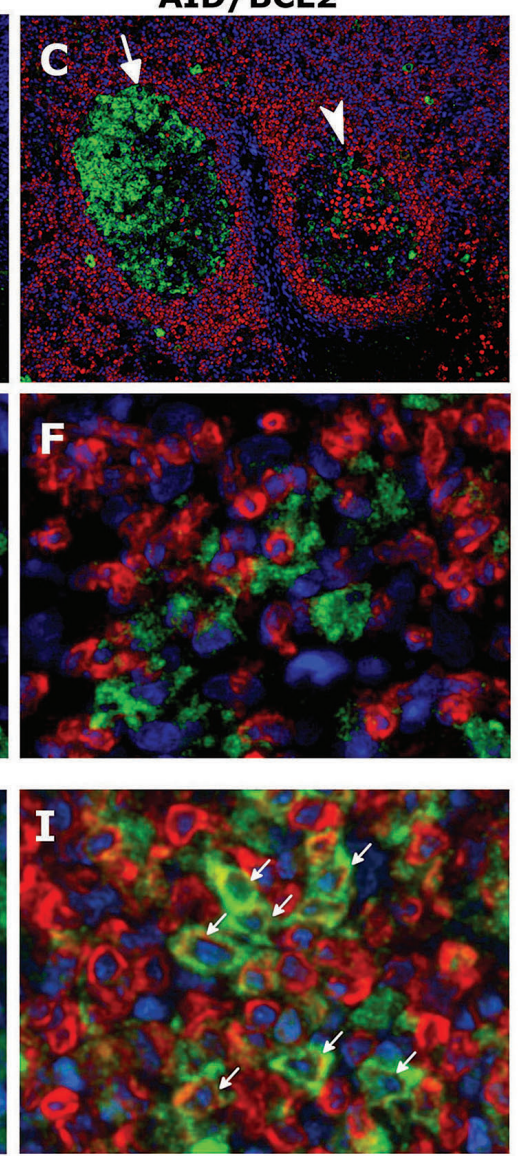

Figure 2. Immunofluorescent AID/BCL2 double staining. (A-C) Follicle (arrowhead) involved by in situ follicular neoplasia (ISFN) with BCL2 staining red cells (A) and few activation-induced cytidine deaminase (AID) staining green cells (B) shows lack of AID/BCL2 double staining cells( C). A nearby reactive follicle (arrow) is negative for BCL2 (A), positive for AID (B) and shows lack of AID/BCL2 double staining cells (C). (D-F) High magnification of the ISFN follicle showing BCL2 (D) positive cells (red), fewer AID (E) staining cells (green), and absence of AID/BCL2 double staining cells (F). (G-I) High magnification of a follicle from lymph node with FL: BCL2 (red) is diffusely positive in lymphoma cells $(G)$ and AID (green) is positive in scattered lymphoma cells (H). AID/BCL2 double staining highlights many dual positive (arrows) lymphoma cells (I).

tive GC cells (Figure 1). In order to confirm this impression, we then performed AID/BCL2 double immunofluorescent (IF) staining on 16 ISFN cases. Double IF staining showed lack of AID expression in strongly BCL2 expressing cells in the ISFN follicles in all the cases (Figure 2). Thus, all cases were considered AID-negative. Internal positive controls (nearby reactive follicles and cells within the same follicle) stained appropriately (BCL2-, $\mathrm{AID}^{+}$). In order to compare the AID expression pattern seen in ISFN to that of typical systemic nodal FL, we also evaluated 15 cases of low grade FL for AID expression using double IF from a tissue microarray constructed during the same time period. Seven of $15(47 \%)$ low grade FL cases demonstrated co-expression of BCL2 and AID in 10\% or more of neoplastic cells within follicles on double IF staining (Figure 2). This difference between systemic nodal FL and ISFN was statistically significant $(P<0.001)$. We could not evaluate $A I D$ expression in three cases with manifest FL at other time points/sites due to the lack of availability of these specimens. Given the known correlation between AID mRNA and protein levels by $\mathrm{IHC}^{6}{ }^{6}$ we further verified the lack of AID expression at mRNA level on two cases each of ISFN and FL by performing RNAscope in situ hybridization. The findings in ISFN were similar to both the IHC and double IF results, confirming the absence (or extremely low levels) of AID in ISFN follicles, compared to manifest FL (Figure 3).
Methodological details are presented in the accompanying Online Supplementary Appendix.

ISFN is an indolent condition with a very low risk $(<5 \%)$ of progression to FL. ${ }^{8}$ The ISFN cells carry a $\mathrm{t}(14 ; 18)$ (q32; $\mathrm{q} 21$ ), similar to usual-type FL. ${ }^{9}$ This abnormality leads to constitutive overexpression of BCL2, inhibition of apoptosis and accumulation of inappropriately rescued $B$ cells with a prolonged life span. This event is believed to be the first genetic hit in the natural history of FL pathogenesis, but additional genetic hits are required for progression to malignant follicular lymphoma. ${ }^{10}$ ISFN carries few secondary genetic changes. ${ }^{9}$ In contrast, secondary genetic alterations are found in $70-90 \%$ of FL at initial diagnosis in addition to the $\mathrm{t}(14 ; 18) / I G H-B C L 2$ fusion. ${ }^{11}$ AID expression is a marker of the germinal center reaction. The additional genetic hits in follicular lymphoma B cells are postulated to be facilitated by AID, contributing to genomic instability. ${ }^{10} \mathrm{~A}$ subset of FL cases (25-100\%) has been shown to express AID. ${ }^{3,45}$ We confirm this in our study by showing that approximately half of the low grade FL cases expressed high level AID in neoplastic follicles by double IF. In our study, none of the ISFN cases expressed detectable AID in BCL2 intensely positive neoplastic cells in GC. The absence of detectable AID in IFSN may be related to greater genetic stability and its generally benign behavior. We cannot exclude very low levels of AID expression, 


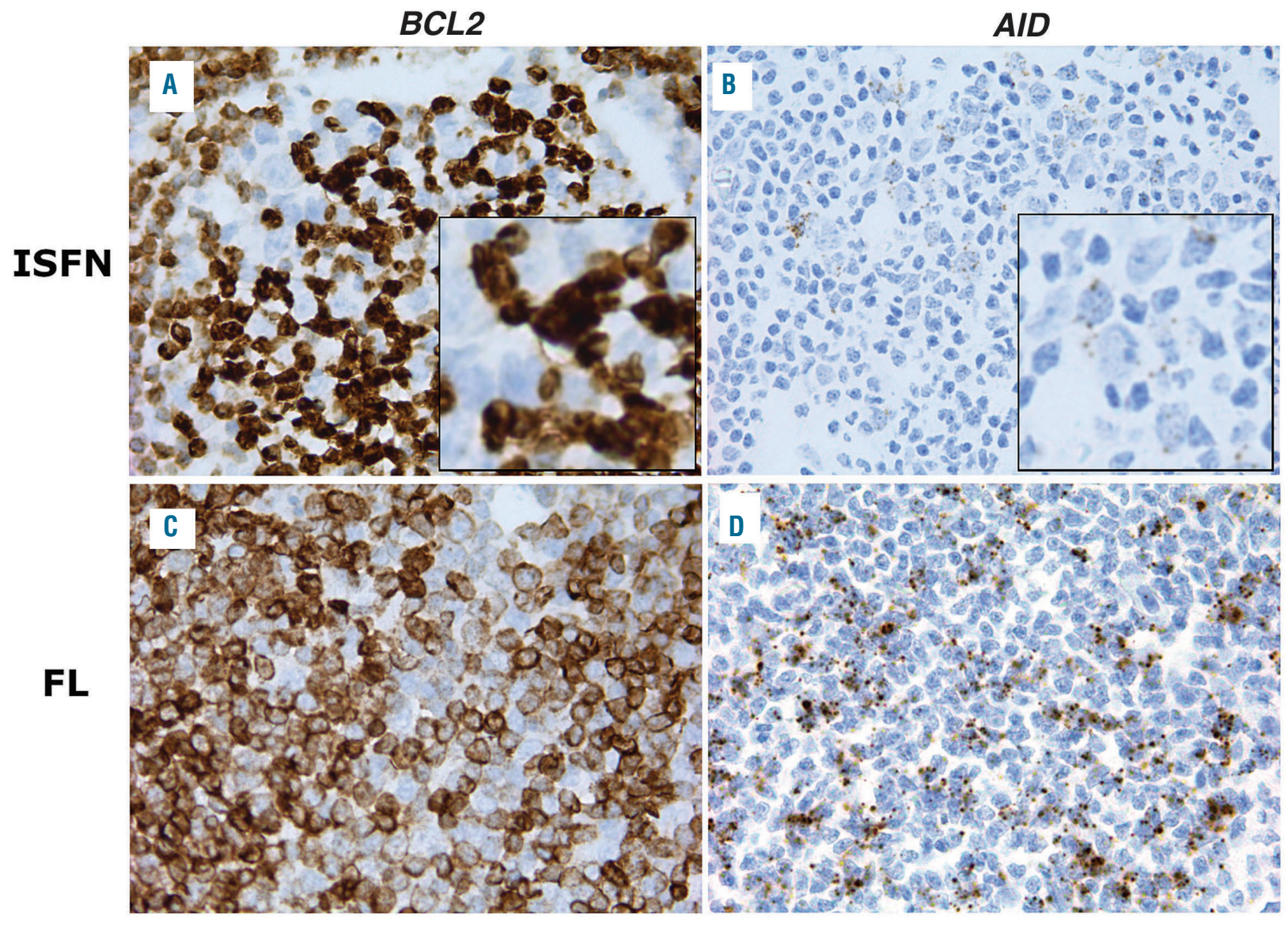

Figure 3. RNAscope, in situ hybridization for AID in in situ follicular neoplasia (results in punctate staining). (A-B) Follicle involved by in situ follicular neoplasia (ISFN) - strongly positive for BCL2 messenger RNA (mRNA) (A) and negative for activation-induced cytidine deaminase (AID) mRNA (B). The few AID positive cells are residual benign germinal center cells which are larger than the small cleaved ISFN cells. Insets are from exactly the same field on serial section. (C-D) Follicular lymphoma- positive for both BCL2 (C) and AID mRNA (D).

below the technical limits of our assays. Indeed, it has been shown that levels of AID in non-isotype switched FL cells correlate with IGHV mutations and aberrant SHM in BCL6. Interestingly, circulating follicular lymphoma-like and ISFN cells, by virtue of the "allelic paradox" phenomenon, are IgM-expressing and show much lower levels of genomic alterations compared to overt FL, consistent with the theory of very low or near absent AID activity at this "early lesion" stage."

The AID expression results in our study are similar to the findings described by Katsuyoshi et al. in duodenaltype FL. ${ }^{7}$ All 17 studied cases of duodenal-type FL lacked expression of AID. Interestingly, a clonal relationship has been proven in a recent case report of ISFN and duodenal-type FL in the same patient. ${ }^{12}$ Our study further demonstrates commonalities between these two entities and reinforce the hypothesis that these two entities might represent different tissue manifestations of a single precursor lesion. ${ }^{12}$ Despite the lack of AID expression, duodenal-type FL B cells have been shown to be at the memory B-cell stage with somatic and ongoing mutations, which later was suggested to be a consequence of BACH2 expression in tumor cells. ${ }^{7,13}$ This may be worth exploring given copy number gains of the $\mathrm{BACH} 2$ locus in ISFN cells. ${ }^{9}$ Interestingly, there is emerging evidence of ongoing IGH SHM in ISFN as recently shown by Kosmidis et al. ${ }^{14}$ Ongoing SHM in ISFN despite the absence of AID expression is not without precedent in typical FL. Ongoing mutations and intraclonal hetero- geneity was detected in AID-negative FL samples, albeit at significantly lower levels than AID-positive cases. ${ }^{4}$ The regulation of AID expression is complex, involving transcription, posttranscription, and posttranslational mechanisms. ${ }^{15}$ This provides for many avenues to explore the mechanism behind ongoing mutations, mechanism for downregulated AID, and the global genetic profile of ISFN that will enhance our understanding of this entity.

Tanu Goyal, Sarah L. Ondrejka, Juraj Bodo, Lisa Durkin and Eric D. Hsi

Cleveland Clinic Robert J. Tomsich Pathology and Laboratory Medicine Institute, Cleveland, OH, USA

Correspondence: ERIC D.HSI - hsie@ccf.org

doi:10.3324/haematol.2020.249342

Disclosures: EDS has received the following support, unrelated to the submitted work: research sponsorship from Abbvie, Eli LIlly as well as honoraria from Jazz, Seattle Genetics and Miltenyi; all other authors have no conflicats of interest to disclose.

Contributions: EDH, TG, SLO prepared the manuscript, JB and $L D$ performed the laboratory work, EDH coordinated reseach.

\section{References}

1. Gu X, Shivarov V, Strout MP. The role of activation-induced cytidine deaminase in lymphomagenesis. Curr Opin Hematol. 2012; 19(4):292-298.

2. Yamane A, Resch W, Kuo N, et al. Deep-sequencing identification of the genomic targets of the cytidine deaminase AID and its cofactor 
RPA in B lymphocytes. Nat Immunol. 2011;12(1):62-69.

3. Smit LA, BendeR, Aten J, Guikema JEJ, Aarts WM, Van Noesel CJM Expression of activation-induced cytidine deaminase is confined to B-cell non-Hodgkin's lymphomas of germinal-center phenotype. Cancer Res. 2003;63(14):3894-3898.

4. Hardianti MS, Tatsumi E, Syampurnawati M, et al. Activationinduced cytidine deaminase expression in follicular lymphoma: association between AID expression and ongoing mutation in FL. Leukemia. 2004;18(4):826-831

5. Greeve J, Philipsen A, Krause K, et al. Expression of activationinduced cytidine deaminase in human B-cell non-hodgkin lymphomas. Blood. 2003;101(9):3574-3580.

6. Shrerer F, Navarrete MA, Bertinetti-Lapatki C, Boehm J, SchmittGraeff A, Veelken H. Isotype-switched follicular lymphoma displays dissociation between activation-induced cytidine deaminase expression and somatic hypermutation. Leuk Lymphoma. 2016; 57(1):151 160

7. Takata K, Sato Y, Nakamura N, et al. Duodenal and nodal follicular lymphomas are distinct: the former lacks activation-induced cytidine deaminase and follicular dendritic cells despite ongoing somatic hypermutations. Mod Pathol. 2009;22(7):940-949.

8. Jegalian AG, Sato Y, Nakamura N, et al. Follicular lymphoma in situ: Clinical implications and comparisons with partial involvement by follicular lymphoma. Blood. 2011;118(11):2976-2984

9. Mamessier E, Song JY, Eberle FC, et al. Early lesions of follicular lymphoma: A genetic perspective. Haematologica 2014;99(3):481-488.

10. Ott G, Rosenwald A. Molecular pathogenesis of follicular lymphoma. Haematologica 2008 Dec;93(12):1773-6.

11. Schmidt J, Salaverria I, Haake A, et al. Increasing genomic and epigenomic complexity in the clonal evolution from in situ to manifest t(14;18)-positive follicular lymphoma. Leukemia. 2014;28(5):11031112 .

12. Nann D, Bonzheim I, Müller I, et al. Clonally related duodenal-type follicular lymphoma and in situ follicular neoplasia. Haematologica. 2019;104(11):e537-e539.

13. Takata K, SatoY, Nakamura N, et al. Duodenal follicular lymphoma lacks AID but expresses BACH2 and has memory B-cell characteristics. Mod Pathol. 2013;26(1):22-31.

14. Kosmidis P, Bonzheim I, Dufke C, et al. Next generation sequencing of the clonal IGH rearrangement detects ongoing mutations and interfollicular trafficking in in situ follicular neoplasia. PLoS One. 2017;12(6):e0178503.

15. Lou Y, Liu Y, Wu L, et al. CUL7 E3 ubiquitin ligase mediates the recombination in $\mathrm{B}$ lymphocytes deaminase and regulates the Ig class switch degradation of activation-induced cytidine. J Immunol. 2019;203(1):269-281 